\title{
Exploring the Association Between Infectious Diarrheal Diseases and Sea Surface Temperatures - Coastal Areas of China, 2009-2018
}

\author{
Min Xu' ${ }^{1}$ Chunxiang Cao ${ }^{1,2, *} ;$ Heyi Guo ${ }^{1,2} ;$ Yiyu Chen ${ }^{1,2} ;$ Zhongwei Jia $^{3}$
}

\section{Summary}

What is already known about this topic?

Coastal areas of China have a higher reported incidence of other infectious diarrheal diseases (OIDD; excluding cholera, dysentery, typhoid, and paratyphoid) than inland areas of China.

What is added by this report?

The incidence of OIDD in high latitude coastal provincial-level administrative divisions (PLADs) near Bohai Sea was positively correlated with sea surface temperatures (SSTs), while in coastal PLADs near the South China Sea was negatively correlated.

What are the implications for public health practice?

The marine environmental risk factors acquired by remote sensing provide a new way for diseases surveillance and early warning. SSTs can be employed as predictor of OIDD in some coastal areas in China.

Infectious diarrhea is an important public health problem worldwide (1). As a type of common and important infectious disease, it poses a serious threat to human health and was ranked as the leading cause of death among people of all ages, especially young children in developing countries (2). Other infectious diarrheal diseases (OIDD) refer to a group of intestinal infectious diseases with diarrhea that exclude cholera, bacterial and amoebic dysentery, typhoid, and paratyphoid. Spatial analysis and Pearson's correlation was employed to explore the association between the monthly OIDD incidence of the each coastal provincial-level administrative divisions (PLADs) and the average monthly sea surface temperature (SST) of its nearby offshore based on ten years data of China from 2009 to 2018. The results showed that the incidence of OIDD in coastal PLADs was higher than that of inland PLADs in China; coastal PLADs of high latitude areas near Bohai Sea had significant positive correlations with the SSTs, but those of low latitude areas near South China Sea were negatively correlated with the SSTs. SSTs can be a potential predictor of OIDD in some coastal areas in China.

Meteorological conditions have been confirmed to impact pathogen exposures, in particular those associated with waterborne transmission. Several epidemiological studies used time series analysis to show that temperature or precipitation factors had a strong triggering effect on diarrhea (3). Extreme rainfall caused floods may contaminate drinking water by flushing diarrhea-causing pathogens from pastures and dwellings into drinking water supplies (4). High ambient temperatures can further promote diarrhea transmission by enhancing pathogen replication rates or by changing water usage behaviors and hygiene and sanitation practices (5). The ocean is the largest reservoir of viruses and bacteria globally. The marine environmental parameters including sea surface temperature, sea surface height anomaly and sea chlorophyll concentration are potential predictors of many infectious diseases such as cholera to coastal regions (6). SSTs in the central equatorial Pacific Ocean have been proved to be linked to diarrhea outbreaks to the many Asian and South American countries including Bangladesh, Peru, and Japan (5). While the association between meteorological factors and diarrhea incidence has been well documented in China, limited attention has been directed at whether the marine environmental factors especially the SST have an influence on the incidence of OIDD in coastal areas of China.

Ten years monthly-recorded OIDD data of China from 2009 to 2018 were extracted from the Datacenter of China Public Health Science. This database included all data since the initiation of network reporting system, which were the number of cases, incidence by PLAD. Only the diarrhea cases confirmed clinically or by laboratory tests, including microscopic examination and biochemical identification, were included in the database. SSTs data of the same period were acquired from NASA'S Jet Propulsion Laboratory (http://podaac.jpl.nasa.gov). The data were produced 
using the satellite images from the National Oceanographic and Atmospheric Administration Advanced Very High Resolution Radiometer (AVHRR). The SSTs data used in the study were monthly AVHRR Oceans Pathfinder SST data with a spatial resolution of $4 \mathrm{~km}$.

Descriptive statistics methods were used to describe the study variables in coastal PLADs of China. ArcGIS software (version 10.2, ESRI, Redlands, USA) was employed for the spatialization of the OIDD incidence data. Meanwhile, Pearson's correlation analysis was conducted to assess the associations between SSTs and OIDD incidence and a two-tailed test of significance was used. Offshore China was divided into four seas including Bohai Sea, Yellow Sea, East China Sea, and South China Sea. The 31 PLADs of the mainland of China were divided into 11 coastal PLADs and 20 non-coastal PLADs. The coastal PLADs near the Bohai Sea include Liaoning, Hebei, Tianjin, and Shandong; near the Yellow Sea include Liaoning, Shandong, and Jiangsu; near the East China Sea include Shanghai, Jiangsu, and Zhejiang; near the South China Sea include Fujian, Guangdong, Hainan, and Guangxi.

The total cases and monthly OIDD incidence of each PLAD in the mainland of China was shown in Table 1. The average incidence of OIDD in coastal PLADs was significantly higher than that in inland PLADs. A total of 9,527,747 OIDD cases were reported in China from 2009 to 2018, including $5,124,303$ cases in the 11 coastal PLADs that accounted for $54 \%$ of the total cases. The average monthly incidence in China was 5.817/100,000. While the monthly incidence in coastal PLADs was higher than the average, which reached 7.230/100,000. Tianjin Municipality had the highest incidence of monthly OIDD, which was 19.846/100,000. Because the consultation rate of OIDD in China was estimated to be between $50 \%$ to $80 \%$, some cases were missed, and the true incidence was higher than the reported incidence.

The dynamic curve of monthly OIDD incidence in coastal PLADs and China from 2009 to 2018 was shown in Figure 1. The data exhibit a clear seasonality with the outbreaks concentrated in summer and winter. The monthly OIDD incidences between 2009 and 2018 in coastal PLADs were always higher than that in inland PLADs all the year around. The time series monthly SSTs extracted from the four offshore areas vary greatly from each other. The average monthly SST in Bohai Sea was $13.2{ }^{\circ} \mathrm{C}$ and varied
TABLE 1. The total cases and monthly OIDD incidence of each PLAD in the mainland of China from 2009 to 2018.

\begin{tabular}{|c|c|c|c|}
\hline Areas & PLADs & $\begin{array}{c}\text { Total OIDD } \\
\text { cases }\end{array}$ & $\begin{array}{c}\text { OIDD incidence } \\
(1 / 100,000)\end{array}$ \\
\hline \multirow{11}{*}{$\begin{array}{l}\text { Coastal } \\
\text { PLADs }\end{array}$} & Tianjin & 370,781 & 19.846 \\
\hline & Zhejiang & $1,059,099$ & 15.602 \\
\hline & Guangdong & $1,334,039$ & 9.953 \\
\hline & Guangxi & 529,972 & 9.041 \\
\hline & Hebei & 535,975 & 5.940 \\
\hline & Fujian & 255,043 & 5.434 \\
\hline & Shandong & 603,132 & 5.023 \\
\hline & Liaoning & 186,186 & 3.551 \\
\hline & Hainan & 39,326 & 3.270 \\
\hline & Shanghai & 52,878 & 1.822 \\
\hline & Jiangsu & 157,872 & 1.638 \\
\hline \multirow{20}{*}{ Inland PLADs } & Beijing & 437,431 & 16.793 \\
\hline & Ningxia & 87,545 & 10.701 \\
\hline & Anhui & 738,954 & 9.845 \\
\hline & Chongqing & 317,852 & 8.613 \\
\hline & Jiangxi & 310,117 & 5.591 \\
\hline & Xinjiang & 154,375 & 5.262 \\
\hline & Shaanxi & 241,495 & 5.247 \\
\hline & Qinghai & 35,155 & 4.896 \\
\hline & Hubei & 334,722 & 4.726 \\
\hline & Henan & 504,081 & 4.394 \\
\hline & Gansu & 118,912 & 3.774 \\
\hline & Shanxi & 161,360 & 3.632 \\
\hline & Hunan & 270,434 & 3.285 \\
\hline & Sichuan & 323,674 & 3.249 \\
\hline & Yunnan & 173,563 & 3.013 \\
\hline & Guizhou & 90,778 & 2.113 \\
\hline & $\begin{array}{c}\text { Inner } \\
\text { Mongolia }\end{array}$ & 35,157 & 1.159 \\
\hline & Heilongjiang & 50,075 & 1.101 \\
\hline & Jilin & 17,022 & 0.522 \\
\hline & Xizang (Tibet) & 742 & 0.200 \\
\hline
\end{tabular}

Abbreviations: OIDD=other infectious diarrheal diseases;

PLADs=provincial-level administrative divisions.

from $1{ }^{\circ} \mathrm{C}$ to $27{ }^{\circ} \mathrm{C}$; the characteristics of SST in Yellow Sea were similar to that of the Bohai Sea; the average value of SSTs in Yellow Sea was $15.3{ }^{\circ} \mathrm{C}$ and it varied from $5{ }^{\circ} \mathrm{C}$ to $28{ }^{\circ} \mathrm{C}$, both of which had seasonality. While average monthly SST in the South China Sea was $27.9{ }^{\circ} \mathrm{C}$ and varied only from $25{ }^{\circ} \mathrm{C}$ to $30{ }^{\circ} \mathrm{C}$. In the East China Sea, the average monthly SST was $22.2{ }^{\circ} \mathrm{C}$ and varied only from $16{ }^{\circ} \mathrm{C}$ to $30{ }^{\circ} \mathrm{C}$.

Pearson's correlation coefficient was employed to analyze the association between the monthly OIDD 
incidence of each coastal PLAD and the average monthly SST of its adjacent offshore sea. Marine environmental factors may have a delayed effect on the OIDD outbreaks, so one-month lag effects for SST were created in the study. The correlation analysis was used for both the current and one-month lag OIDD with the SST. As shown in Table 2, the PLADs of Liaoning, Hebei, Tianjin, Shandong, and Shanghai had significant correlations with the SSTs. The monthly OIDD incidences in Fujian and Guangdong were significantly negative correlated with the SSTs. However, Jiangsu, Zhejiang, Hainan, and Guangxi had little or no significant correlation with SSTs.

\section{DISCUSSION}

Our findings confirm the average OIDD incidence in coastal PLADs was higher than in inland areas of China and demonstrated an association between coastal SSTs and local OIDD incidence. Specifically, we found that coastal PLADs of high latitude areas near Bohai Sea had significant positive correlations with the SSTs, but those of low latitude areas near South China Sea were negatively correlated with the SSTs. No matter in coastal PLADs or inland areas, reported cases mostly occurred in summer and winter, and the incidence dynamic curve showed bimodal fluctuation.

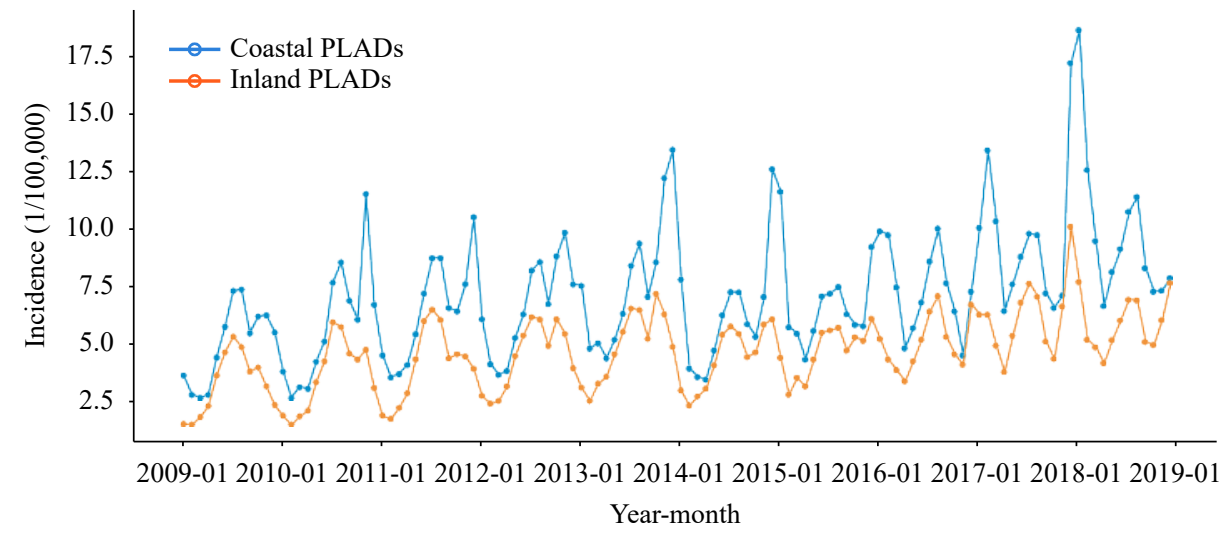

FIGURE 1. The dynamiccurve of monthly OIDD incidence in coastal and inland PLADs of mainland China from 2009 to 2018. Abbreviations: OIDD=other infectious diarrheal diseases; PLADs=provincial-level administrative divisions.

TABLE 2. Pearson's correlation analysis between SSTs and OIDD in coastal PLADs of mainland China.

\begin{tabular}{|c|c|c|c|}
\hline Coastal seas & Coastal PLADs & $\begin{array}{c}\text { Pearson's correlation coefficient between } \\
\text { SSTs and OIDD incidence }\end{array}$ & $\begin{array}{c}\text { Pearson's correlation coefficient between SSTs } \\
\text { and one month lag OIDD incidence }\end{array}$ \\
\hline \multirow{4}{*}{ Bohai Sea } & Liaoning & $0.674^{*}$ & $0.451^{*}$ \\
\hline & Hebei & $0.712^{*}$ & $0.583^{*}$ \\
\hline & Tianjin & $0.834^{*}$ & $0.578^{*}$ \\
\hline & Shandong & $0.447^{*}$ & $0.343^{*}$ \\
\hline \multirow{3}{*}{ Yellow Sea } & Liaoning & $0.679^{*}$ & $0.413^{*}$ \\
\hline & Shandong & $0.442^{*}$ & $0.306^{*}$ \\
\hline & Jiangsu & $0.024^{\dagger}$ & $0.128^{\dagger}$ \\
\hline \multirow{4}{*}{ East China Sea } & Jiangsu & $0.044^{\dagger}$ & $0.154^{\dagger}$ \\
\hline & Zhejiang & $0.193^{\pi}$ & $0.327^{*}$ \\
\hline & Shanghai & $0.579^{*}$ & $0.322^{*}$ \\
\hline & Fujian & $-0.385^{*}$ & $-0.120^{\dagger}$ \\
\hline \multirow{3}{*}{ South China Sea } & Guangdong & $-0.457^{*}$ & $-0.131^{\dagger}$ \\
\hline & Hainan & $-0.187^{\pi}$ & $-0.081^{\dagger}$ \\
\hline & Guangxi & $-0.184^{\pi}$ & $-0.096^{\dagger}$ \\
\hline
\end{tabular}

Abbreviations: SSTs=Sea surface temperatures; OIDD=other infectious diarrheal diseases; PLADs=provincial-level administrative divisions. * Significant correlation at 0.05 level.

${ }^{\dagger}$ No significant correlation.

"Significant correlation at 0.01 level. 
The incidence of OIDD in coastal PLADs from 2009 to 2018 was higher than that of inland PLADs. A possible reason should be related to the impact of marine environmental factors. Although the underlying mechanisms by which marine environmental factors influence infectious diarrheal diseases have not been fully clarified, these marine environments, such as SST, can impact the production or transmission of some pathogens in coastal areas. Pathogens may spread quickly through the contaminated water after flooding. In addition, the consumption and improper preservation of seafood in coastal PLADs may also be important factors affecting the OIDD incidence.

There is a spatial heterogeneity of OIDD distribution in China that the incidence of OIDD in different latitude coastal PLADs varies from each other. This further reinforced results of a former study that suggested the difference of the health effects of temperature in different regions is related to latitude. The effect of temperature on OIDD could be modified by latitude ( 7 ). The risk of OIDD was higher in high latitude areas at low temperatures, suggesting that high latitude areas were vulnerable areas in cold seasons (8). For these areas, we should improve the public's awareness of OIDD prevention and medical treatment and enhance the supply of medical resources. Many former studies have shown that high temperatures may increase the risk of infectious diarrhea by affecting pathogen activity, accelerating food decomposition, and increasing drinking water demand (9). However, some areas have shown that infectious diarrhea is negatively correlated with temperature.

This study was subject to some limitations. First, the SSTs were extracted from satellite retrieved products, which itself is subject to a certain level of error. Second, the mechanism of the impact of the marine environment on the incidence of diarrhea is not clear yet, and the optimal impact of the marine environment on it is unknown. Therefore, we only calculate the average value of the sea surface temperature in each offshore sea area for analysis of the association with OIDD. Finally, sea surface temperature plays an important role in the survival and reproduction of infectious diarrhea in host environments a side from humans and will affect the transmission speed of diarrhea, so there are still some other marine variables that maybe impact on the incidence of OIDD in coastal PLADs. Sea surface height can reflect the characteristics of climate change such as drought stress, water surface rise, and flooding caused by El Niño events. Seawater salinity and seawater chlorophyll concentration are important factors for the transmission and reproduction of pathogenic microorganisms in coastal PLADs. All these impact factors can be predictors for OIDD in coastal PLADs of China.

Conflicts of interest: No conflicts of interest.

Acknowledgments: The Data-center of China Public Health Science.

Funding: Supported by the National Natural Science Foundation of China (grant number: 41971394) and Forest and grass science and technology innovation development and research project of the State Forestry and Grassland Administration of China (grant number: 2020132108).

doi: $10.46234 / \mathrm{ccdcw} 2022.023$

\# Corresponding author: Chunxiang Cao, caocx@aircas.ac.cn.

\begin{abstract}
State Key Laboratory of Remote Sensing Science, Aerospace Information Research Institute, Chinese Academy of Sciences, Beijing, China; ${ }^{2}$ University of Chinese Academy of Sciences, Beijing, China; ${ }^{3}$ School of Public Health, Peking University, Beijing, China.
\end{abstract}

Submitted: November 25, 2021; Accepted: January 07, 2022

\section{REFERENCES}

1. Liu ZD, Zhang FF, Zhang Y, Li J, Liu XN, Ding GY. Association between floods and infectious diarrhea and their effect modifiers in Hunan province, China: a two-stage model. Sci Total Environ 2018;626:630 - 7. http://dx.doi.org/10.1016/j.scitotenv.2018.01.130.

2. Wang H, Di B, Zhang TJ, Lu Y, Chen C, Wang DH, et al. Association of meteorological factors with infectious diarrhea incidence in Guangzhou, southern China: a time-series study (2006-2017). Sci Total Environ 2019;672:7 - 15. http://dx.doi.org/10.1016/j.scitotenv.2019. 03.330 .

3. Liu L, Johnson HL, Cousens S, Perin J, Scott S, Lawn JE, et al. Global, regional, and national causes of child mortality: an updated systematic analysis for 2010 with time trends since 2000. Lancet 2012;379(9832):2151 - 61. http://dx.doi.org/10.1016/S0140-6736(12) 60560-1.

4. Zhang N, Song DD, Zhang J, Liao WM, Miao KC, Zhong S, et al. The impact of the 2016 flood event in Anhui province, China on infectious diarrhea disease: an interrupted time-series study. Environ Int 2019;127:801 - 9. http://dx.doi.org/10.1016/j.envint.2019.03.063.

5. Heaney AK, Shaman J, Alexander KA. El Niño-southern oscillation and under-5 diarrhea in Botswana. Nat Commun 2019;10(1):5798. http:// dx.doi.org/10.1038/s41467-019-13584-6.

6. Xu M, Cao CX, Wang DC, Kan B, Xu YF, Ni XL, et al. Environmental factor analysis of cholera in China using remote sensing and geographical information systems. Epidemiol Infect 2016;144:940 - 51. http://dx.doi. org/10.1017/S095026881500223X.

7. Wang HT, Liu ZD, Xiang JJ, Tong MX, Lao JH, Liu YY, et al. Effect of ambient temperatures on category $\mathrm{C}$ notifiable infectious diarrhea in China: an analysis of national surveillance data. Sci Total Environ 2021;759:143557. http://dx.doi.org/10.1016/j.scitotenv.2020.143557.

8. Wang HT, Liu ZD, Lao JH, Zhao Z, Jiang BF. Lag effect and influencing factors of temperature on other infectious diarrhea in Zhejiang province. Chin J Epidemiol 2019;40(8):960 - 4. http://dx.doi. org/10.3760/cma.j.issn.0254-6450.2019.08.016. (In Chinese).

9. Zhang Y, Bi P, Hiller JE, Sun YW, Ryan P. Climate variations and bacillary dysentery in northern and southern cities of China. J Infect 2007;55:194 - 200. http://dx.doi.org/10.1016/j.jinf.2006.12.002. 